\title{
Protein crystals and charged surfaces: interactions and heterogeneous nucleation
}

\author{
R. P. Sear ${ }^{1, \text { * }}$ \\ ${ }^{1}$ Department of Physics, University of Surrey, Guildford, Surrey GU2 7XH, United Kingdom
}

\begin{abstract}
As proteins typically have charges of around 10, they will interact strongly with charged surfaces. We calculate the electrostatic contribution to the interaction of crystals of protein with charged surfaces. The surfaces repel like-charged crystals and attract oppositely-charged crystals, with free energies which can be easily several kT per protein molecule brought into contact with the surface. This means that oppositely charged surfaces can act as a nucleant, they can induce nucleation of a protein crystal by lowering the free energy barrier to heterogeneous nucleation of the crystal from a dilute solution.
\end{abstract}

\section{INTRODUCTION}

Here we will consider the interaction between charged surfaces and protein crystals. Proteins are themselves charged and so we would expect them to interact strongly with a surface that is charged. Using the PoissonBoltzmann equation we find that indeed protein crystals are attracted by oppositely charged surfaces and repelled by surfaces with charges of the same sign. In itself this is hardly surprising but with quite simple calculations we quantify this attraction and repulsion. We show that easily accessible surface charge densities of the opposite sign to that of the protein molecules, are able to greatly favour heterogeneous nucleation at the surface by reducing the free energy of a nucleus at the surface by several $k_{\mathrm{B}} T$ per protein molecule at the surface. Controlled heterogeneous nucleation is vital to the production of protein crystals, which are required for structure determination via $\mathrm{X}$-ray crystallography.

At a first-order phase transition, such as the crystallisation of a protein from solution, the transition starts with nucleation [1, 2]. Protein crystallisation is the "main bottleneck" [3] in the determination of the threedimensional structure of proteins. Determining this structure is crucial for understanding what a protein does and how it does it. Protein crystallographers wish to: i) induce nucleation in the relatively weakly supersaturated solutions, within which protein crystals grow slowly and so incorporate few defects, and ii) control heterogeneous nucleation, in particular have nucleation occurring only at specific locations on a surface. If crystals only nucleate on widely separated patches on a surface then the growth of one crystal will not interfere with and limit the growth of another crystal.

See for example Refs. [4, 5] for experimental work on adding solid surfaces to protein solutions to induce nucleation, and see Refs. [6, 7] for experimental work on patterned surfaces, which shows that protein crystals can be made to grow preferentially on specified patches of a surface. In Refs. 6, 7] a patterned surface is used in which patches of the surface are one form of doped $\mathrm{Si}$

*Electronic address: r.sear@surrey.ac.uk and the rest is a different form. The protein nucleates preferentially on one of these forms and so by controlling where this form is found in the pattern, the crystals can be made to nucleate and grow far from each other, facilitating the formation of large crystals. Here we show that a pattern of two different surface types of opposite charges can produce sufficiently large differences in surface free energy to make heterogeneous nucleation much easier on one surface than on the other.

We note that here we only consider the effects of charge interactions, screened by a 1:1 electrolyte. Other interactions, such as short-range specific interactions between the protein molecules and surface are known to be important, see the review of Ostuni et al. [8]. When these interactions are important, our calculations, which neglect them, will only yield estimates of the variation with 1:1 salt concentration, of the interaction free energy of a protein crystal or crystalline nucleus with a surface. However, experimental systems do exist with high, variable charges and highly hydrophilic surfaces. These highly hydrophilic surfaces will have only weak non-electrostatic interactions with the proteins, they also has the advantage of minimising the problem of protein denaturation.

One example is a highly charged self-assembled monolayer, such as has been shown to adsorb polyelectrolytes 91, another is a membrane containing charged lipids [10, 11]. Both of these systems should be well described by the theory presented here. There is a great deal of work on the association of proteins and DNA with oppositely charged lipid membranes, Refs. 10, 11] are just two examples of a large literature. As far as the author is aware, there has been no experimental study of adsorption of protein on a highly charged, highly hydrophilic, self-assembled monolayer, but such a system would offer the opportunity to study the effect of electrostatics with minimal interference from other interactions. This is true both for the adsorption of single proteins and of protein crystals.

Our calculations will all be for infinite planar interfaces, despite the fact that our primary interest is in the nuclei of protein crystals against charged surfaces, not bulk crystals against surfaces. Although, nuclei are small, 10s of protein molecules, the part of the free energy which scales with the contact area between the nucleus and the surface will still dominate the edge contribution, 
FIG. 1: A schematic of a nucleus of a protein crystal against a charged surface. The hatched circles are the proteins in the crystal lattice, each with its charge $Q$ marked. The surface is shaded grey and the surface charges, positive in this case, are shown as +'s.

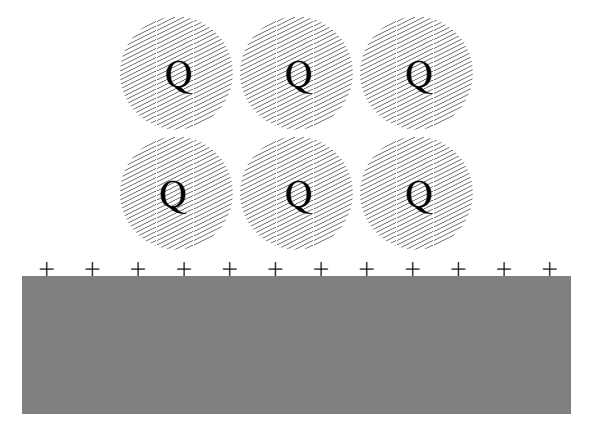

as the Debye length is typically a few nms at most. Thus, we consider only the dominant surface term, considering sub-dominant terms within simple theories of simple generic models is not useful. We will also leave consideration of defects in the surface to later work.

In the next section we calculate the electrostatic contributions to the surface free energy. The third section includes example results and a discussion and the last section is a conclusion.

\section{CALCULATION}

Here we calculate the electrical double layer contributions to the interfacial free energies of solid-salt-solution, solid-protein-crystal and solution-protein-crystal interfaces. The calculation for the solution-protein-crystal interface is just a repeat of that done in Ref. [12] and so will not be described in detail. We will use the model of Ref. 12] for the protein crystal and solution, along with a simple model of a charged solid surface. As we are interested in interfacial free energies it is most convenient to work at constant chemical potential of the salt, and so the appropriate free energy is the grand potential $\Omega$. We will split this grand potential into the part due to the charges on the surface and on the protein molecules, together with the salt and counter ions, $\Omega_{e l}$, and another part which includes the rest of the interactions, $\Omega_{n e l}$,

$$
\Omega=\Omega_{n e l}+\Omega_{e l}
$$

$\Omega_{n e l}$ includes effects such as the dispersion interactions between the protein molecules and the surface, and any short-range interactions between the surfaces of the proteins and the solid surface. We will not calculate $\Omega_{n e l}$, and so will only be able to estimate the absolute values of the interfacial free energies, when $\Omega_{n e l}$ is much smaller than $\Omega_{e l}$, for example with highly charged, highly hydrophilic, surfaces, and highly charged proteins. However, if we assume that $\Omega_{n e l}$ is weakly dependent on the salt concentration, and that the charges on the surface can be varied without varying $\Omega_{n e l}$ significantly, our results will describe the variation in the interfacial free energies with surface charge density and the salt concentration.

Rather than considering a specific protein crystal, we take over the jellium concept from the theory of metals to make a general estimate of the effect of salt on the interfacial tension. We replace the detailed charge density due to the protein molecules by a uniform background charge density $\rho_{p}$, cut off abruptly at the interface. In Ref. 12], Sear and Warren did so for the protein-crystalsalt-solution interface, and this followed on from the work of Warren [13] on the bulk phase behaviour. Here when we calculate the interfacial free energy for a protein crystal at the surface, we will assume the protein crystal is flush against the surface and so the charge density is uniform right up to the surface. Our model of the charged surface of the substrate is that of an infinite charged plane at a fixed charge per unit area $\sigma$. The solution outside the crystal is taken to be sufficiently dilute that we can neglect any protein molecules in it and treat it as a salt solution at a concentration $\rho_{s}$. For definiteness we take the protein to be positively charged so the counterions are negative and the coions are positive; both are monovalent. The surface is in the $x y$ plane at $z=0$, with the solid in the $z<0$ half-space and either a salt solution or a protein crystal in the $z>0$ half-space.

We will use the same notation as in Ref. [12], so we work in units where $e=k_{\mathrm{B}} T=1$. In these units, the Coulomb potential energy $U$ between a pair of elementary charges separated by $r$ is $U=l_{\mathrm{B}} / r$, where $l_{\mathrm{B}}$ is the Bjerrum length, equal to $0.72 \mathrm{~nm}$ in water at room temperature $\left(l_{\mathrm{B}}=e^{2} / 4 \pi \epsilon k_{\mathrm{B}} T\right)$. We assume a constant value of $l_{\mathrm{B}}$, and ignore dielectric effects. The counterion and coion densities are $\rho_{-}(z)$ and $\rho_{+}(z)$, respectively. In the bulk salt solution, both tend to the salt concentration $\rho_{s}$, and inside the protein crystal $\left(\rho_{-}-\rho_{+}\right) \rightarrow \rho_{p}$.

We will use a grand potential, $\Omega_{e l}$, which contains only ideal solution terms for the ions and the associated electric field at the interface. The grand potential $\Omega_{e l}$, is [12],

$$
\Omega_{e l}=\int_{0}^{\infty} d z \omega(z), \quad \omega=\sum_{i= \pm} \rho_{i}\left(\ln \frac{\rho_{i}}{\rho_{s}}-1\right)+\frac{E^{2}}{8 \pi l_{\mathrm{B}}}
$$

The first terms in $\omega$ are the ideal solution terms (the ions share a common chemical potential $\mu_{ \pm}=\ln \rho_{s}$ ). The last term is the electrostatic energy, wherein $E=-d \phi / d z$ is the electric field strength corresponding to an electrostatic potential $\phi$ which satisfies the Poisson equation,

$$
\frac{d^{2} \phi}{d z^{2}}=\left\{\begin{array}{ll}
-4 \pi l_{\mathrm{B}}\left(\rho_{+}-\rho_{-}\right) & \text {salt solution } \\
-4 \pi l_{\mathrm{B}}\left(\rho_{+}-\rho_{-}+\rho_{p}\right) & \text { protein crystal }
\end{array},\right.
$$

The variational principle $\delta \Omega_{e l} / \delta\left[\rho_{ \pm}(z)\right]=0$ applied in this problem yields $\rho_{ \pm}(z)=\rho_{s} \exp [\mp \phi]$. The electrostatic potential then satisfies the Poisson-Boltzmann 
equation,

$$
\frac{d^{2} \phi}{d z^{2}}-\kappa_{s}^{2} \sinh \phi=\left\{\begin{array}{ll}
0 & \text { salt solution } \\
-4 \pi l_{\mathrm{B}} \rho_{p} & \text { protein crystal }
\end{array},\right.
$$

where

$$
\kappa_{s}^{2}=8 \pi l_{\mathrm{B}} \rho_{s}
$$

For our assumption of a planar surface in the $z=0 x y$ plane, with a fixed charge per unit area $\sigma$, the boundary condition on $\phi$ at $z=0$, is that the electric field at the surface

$$
E=-\frac{d \phi}{d z}=\frac{\sigma}{\epsilon}=4 \pi l_{\mathrm{B}} \sigma
$$

The other boundary condition is that $d \phi / d z=0, z \rightarrow \infty$.

\section{A. Surface-salt-solution interface}

This is just the problem addressed by Gouy and Chapman at the beginning of the last century 14]. For simplicity we will only derive the expressions in the regime where the equations may be linearised. Then we will simply state the general result obtained when the equations are not linearised. Linearising the Poisson-Boltzmann equation, Eq. (4), inside the salt solution, and using the boundary conditions, we have

$$
\phi(z)=4 \pi l_{\mathrm{B}} \sigma \kappa_{s}^{-1} \exp \left(-\kappa_{s} z\right) \quad z>0 .
$$

A simple exponential decay from a value at the surface, proportional to the surface charge.

The surface free energy is, by definition, the difference between the actual grand potential per unit area of the surface-salt-solution interface and that it would have if the salt solution continued unperturbed right up to solid surface. We therefore have to calculate the grand potential, Eq. (2), then subtract the grand potential for the bulk solution: Eq. (2) with the actual $\omega(z)$ replaced by its value in the bulk salt solution, $\omega(+\infty)$ [15],

$$
\Delta \gamma_{\mathrm{s}}=\int_{0}^{\infty} d z(\omega(z)-\omega(+\infty))
$$

This is a general expression, in the linear regime we can expand out $\omega$ of Eq. (2) and keeping only the terms up to quadratic order in $\phi$, we have that $\omega=-2 \rho_{s}+2 \rho_{s} \phi^{2}$. The bulk value $\omega(+\infty)=-2 \rho_{s}$, as the potential in the bulk of the salt solution is taken to be zero. The surface free energy is then obtained by substituting these expressions into Eq. (8). Doing this and using Eq. (7), we have

$$
\Delta \gamma_{\mathrm{s}}=2 \pi l_{\mathrm{B}} \sigma^{2} \kappa_{s}^{-1} \quad \text { linear, }
$$

which is positive and proportional to the square of the surface charge and to the screening length, as we might have expected.
If Eq. (4) is not linearised the potential $\phi$ is given by [12, 14] $\phi=2 \ln \left[\left(1+C e^{-\kappa_{s} z}\right) /\left(1-C e^{-\kappa_{s} z}\right)\right]$ where $C$ is determined by the boundary condition Eq. (6) and is given by

$$
C=\frac{\kappa_{s}}{2 \pi l_{\mathrm{B}} \sigma}\left[\sqrt{1+\left(\frac{2 \pi l_{\mathrm{B}} \sigma}{\kappa_{s}}\right)^{2}}-1\right] .
$$

Thus, we can evaluate the surface free energy once we have converted Eq. (8) into an expression in terms of $\phi$ only. Equation (4) in the salt solution can be integrated once with respect to $\phi$,

$$
\left(\frac{d \phi}{d z}\right)^{2}=2 \kappa_{s}^{2}(\cosh \phi-1)
$$

which gives us the electric field term in $\omega$. The other, ideal gas, term can be written in terms of $\rho_{s}$ and $\phi$ using $\rho_{ \pm}(z)=\rho_{s} \exp [\mp \phi]$. Then $\Delta \gamma_{\mathrm{s}}$ is given by the integral

$$
\Delta \gamma_{\mathrm{s}}=2 \rho_{s} \int_{0}^{\infty} d z \phi \sinh \phi
$$

which is straightforward to evaluate numerically.

\section{B. Surface-protein-crystal interface}

As with the surface-salt-solution interface we start with the linear Poisson-Boltzmann equation. Here the potential is that inside the protein crystal so we linearise Eq. (4) and obtain

$$
\frac{d^{2} \phi}{d z^{2}}=\kappa_{s}^{2}\left(\phi-\frac{\rho_{p}}{2 \rho_{s}}\right)
$$

where we used Eq. (5) for $\kappa_{s}$. The second term inside the parentheses is minus the Donnan potential $\phi_{\mathrm{D}}$ inside the protein crystal. The general expression for the Donnan potential is

$$
\sinh \phi_{\mathrm{D}}=\frac{\rho_{p}}{2 \rho_{s}}
$$

which when linearised gives $\phi_{\mathrm{D}}=\rho_{p} /\left(2 \rho_{s}\right)$. Thus, the solution to Eq. (13), with the boundary condition of fixed charge density, Eq. (6), is

$$
\phi(z)=\phi_{\mathrm{D}}+4 \pi l_{\mathrm{B}} \sigma \kappa_{s}^{-1} \exp \left(-\kappa_{s} z\right) \quad z>0 .
$$

In the bulk protein crystal, $\phi=\phi_{\mathrm{D}}$. We expand the $\omega$ of Eq. (2) in powers of $\phi_{\mathrm{D}}$ and keep only the terms up to quadratic order, and find that in the crystal $\omega(-\infty)=$ $-2 \rho_{s}+2 \rho_{s} \phi_{\mathrm{D}}^{2}$ [12]. Performing the same expansion at the interface, we obtain for the excess grand potential at the interface

$$
\omega-\omega(-\infty)=\rho_{s}\left[\phi^{2}-\phi_{\mathrm{D}}^{2}+2\left(\phi-\phi_{\mathrm{D}}\right)^{2}\right],
$$


where we used Eq. (15) to obtain the derivative $d \phi / d z$. The interfacial free energy per unit area $\Delta \gamma_{\mathrm{x}}$ is the integral over all $z$ of the excess grand potential of Eq. (16). It is

$$
\Delta \gamma_{\mathrm{x}}=2 \pi l_{\mathrm{B}} \sigma^{2} \kappa_{s}^{-1}+\sigma \phi_{\mathrm{D}} \quad \text { linear, }
$$

If we do not linearise the Poisson-Boltzmann equation, Eq. (4), we can still integrate it once to obtain

$$
\left(\frac{d \phi}{d z}\right)^{2}=2 \kappa_{s}^{2}\left(\cosh \phi-\cosh \phi_{\mathrm{D}}\right)-8 \pi l_{\mathrm{B}} \rho_{p}\left(\phi-\phi_{\mathrm{D}}\right) .
$$

Combining this with the boundary condition, Eq. (6) we obtain an equation for the potential at the surface $\phi(z=$ $0)$,

$$
\begin{aligned}
& {\left[2 \kappa_{s}^{2}\left(\cosh \phi(z=0)-\cosh \phi_{\mathrm{D}}\right)-8 \pi l_{\mathrm{B}} \rho_{p}\left(\phi(z=0)-\phi_{\mathrm{D}}\right)\right]^{1 / 2}} \\
& = \pm 4 \pi l_{\mathrm{B}} \sigma,
\end{aligned}
$$

which can be solved for $\phi(z=0)$, and then once this is known the profile $\phi(z)$ is readily obtained by numerically integrating Eq. (18). On the right hand side the $+(-)$ sign is taken when $\sigma>0(\sigma<0)$. The interfacial free energy is then obtained from

$$
\Delta \gamma_{\mathrm{x}}=\int_{0}^{\infty} d z(\omega(z)-\omega(-\infty)),
$$

where the grand potential per unit volume in the protein crystal is $\omega(-\infty)=-2 \rho_{s}\left(\cosh \phi_{\mathrm{D}}-\phi_{\mathrm{D}} \sinh \phi_{\mathrm{D}}\right)$. Using $\rho_{ \pm}(z)=\rho_{s} \exp [\mp \phi]$, and Eq. (18) for the electric field term in Eq. (2), we have that the electrical double layer contribution to the free energy of the solid-protein interface is given by

$$
\Delta \gamma_{\mathrm{x}}=2 \rho_{s} \int_{0}^{\infty} d z \phi\left[\sinh \phi-\sinh \phi_{\mathrm{D}}\right] .
$$

\section{Salt-solution-protein-crystal interface}

This interface was the subject of Ref. 12. In the high salt regime where the Poisson-Boltzmann equation can be linearised the electrical double layer contribution to the free energy of the interface between a protein crystal and a salt solution is

$$
\Delta \gamma_{\mathrm{el}}=-\frac{\rho_{s} \phi_{\mathrm{D}}^{2}}{2 \kappa_{s}} \quad \text { linear. }
$$

See Ref. [12] for the full nonlinear calculation.

\section{RESULTS}

We can calculate the free energies of the three interfaces: solid-salt-solution, solid-protein-crystal and saltsolution-protein-crystal. This allows us to see whether
FIG. 2: The electrical double layer contribution to the change in free energy per unit area, times the surface area of the crystal per protein molecule, when a protein crystal is brought from the bulk of the salt solution to the charged surface of a solid, $\Delta l^{2}$, Eq. 23. The solid, dotted, dot-dashed and dashed curves are for charge densities $\sigma e=1,0.1,-0.1$ and $-1 \mathrm{~nm}^{-2}$, respectively. Results are shown for salt concentrations down to $0.025 \mathrm{M}$. In the limit of the salt concentration tending to zero the surface free energies diverge.

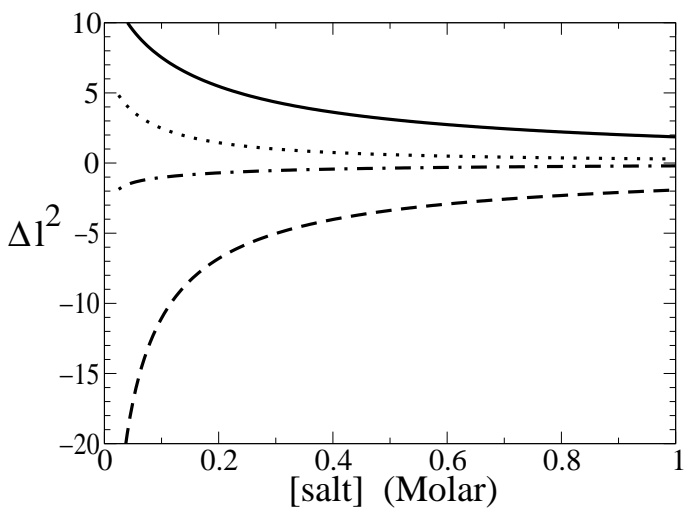

a protein crystallite, such as a nucleus or growing crystallite in solution, is attracted or repelled by the surface. It is attracted if the free energy change on bringing a protein crystal from the bulk solution to the surface is negative and repulsive if the change is positive. When a protein crystallite is brought from the bulk of the solution into contact with the surface two interfaces are destroyed, that between the salt solution and the protein crystal and that between the salt solution and the solid, and one is created, that between the solid and the protein crystal. Thus, the free energy change per unit area of protein crystal brought into contact with the solid is

$$
\Delta=-\Delta \gamma_{\mathrm{s}}-\Delta \gamma_{\mathrm{el}}+\Delta \gamma_{\mathrm{x}}
$$

which in the high salt limit is, using Eqs. (9), (17) and (22),

$$
\Delta=\frac{\rho_{s} \phi_{\mathrm{D}}^{2}}{2 \kappa_{s}}+\sigma \phi_{\mathrm{D}} \quad \text { linear. }
$$

The first term comes from the disappearance of the saltsolution-protein-crystal interface and is positive as the electrical double layer contribution to the interfacial tension of this interface is always negative; see Ref. [12] for a discussion. As $\phi_{\mathrm{D}}$ has the same sign as that of the charges on the protein molecules (here taken to be positive), the second term is positive if the surface and protein molecules have charges of the same sign and negative if the have opposite signs. As we should expect, surfaces repel crystals of proteins with the same charge. They attract crystals of oppositely charged protein molecules, providing that the surface charge $\sigma$ is sufficiently high. 
If the charge density is small or zero then they repel protein crystals due to the free energy cost of destroying the salt-solution-protein-crystal interface.

Equation (24) is obtained by linearising the PoissonBoltzmann equation. We have also solved the full equation and present example results in Figs. 2 and 3 The parameters for the protein are those used previously [12], and were chosen to model lysozyme. This is a small protein with quite a large net charge for its size, whose behaviour in $\mathrm{NaCl}$ solutions has been extensively studied 13, 16, 17, 18, 19. The model used here has been to shown to give the variation with $\mathrm{NaCl}$ concentration of the solubility of lysozyme crystals correctly to within a factor of about 2 , it overpredicts the variation [13, 19]. The agreement between its predictions and the surface force apparatus measurements of Sivasankar et al. 20] of the repulsion between monolayers of the protein streptavidin, is comparable 12. To model lysozyme we took a charge $Q=10[13,17,21$, and a charge density due to the protein $\rho_{p}=0.25 \mathrm{~nm}^{-3}$, obtained by taking the volume per lysozyme molecule in the crystal to be $40 \mathrm{~nm}^{3}$. The surface area of the protein crystal per lysozyme molecule is taken to be $l^{2}=12 \mathrm{~nm}^{2} 12$. For proteins such as lysozyme which have a significant net charge we would expect our predictions to be approximately as accurate as those of earlier predictions of this theory, i.e., predicting the trends correctly but giving numbers which are a factor of 2 or more out. We expect our predictions to be unreliable if the net charge is small, then the interaction between the dipole moment of the protein and the surface may dominate, or if the charge is distributed on the surface in a way which is highly inhomogeneous. If the charge density within the volume within a few Debye lengths of the surface differs greatly from $\rho_{p}$, then our assumption of uniform charge density due to the protein will be poor. See Ref. 12] for further discussion of the assumption that the protein crystal can be modeled by a step-function charge density.

In Fig. 2 we see that for lysozyme and solid surfaces with $\sigma= \pm 1 \mathrm{~nm}^{-2}$, the free energy change of bringing the protein crystal into contact with the solid is substantial unless the salt concentration is around 1M. Even at a salt concentration of $1 \mathrm{M}$, the free energy change is close to $2 k_{\mathrm{B}} T$ per lysozyme molecule at the surface of the protein in contact with the solid surface. Whereas at salt concentrations of about $0.1 \mathrm{M}$ and lower the free energy change is of order $10 k_{\mathrm{B}} T$ per lysozyme molecule. Note however, that we have assumed that the protein crystal is in a salt solution, i.e., a solution with negligible amounts of protein in it. Decreasing the salt concentration increases the solubility of lysozyme 17], and so this assumption will tend to worsen as the salt concentration drops [12, 13. The salt is not only changing the interaction between the protein and the surface but also the interaction in between protein molecules and this needs to be borne in mind.

In Fig. 3 we see that at high salt concentrations, 1M, the free energy change on bringing a crystal into con-
FIG. 3: The electrical double layer contribution to the change in free energy per unit area, times the surface area of the crystal per protein molecule, when a protein crystal is brought from the bulk of the salt solution to the charged surface of a solid, $\Delta l^{2}$, Eq. 23). The solid, dashed and dotted curves are for salt concentrations of $\rho_{s}=0.1,0.5$ and $1 \mathrm{M}$, respectively.

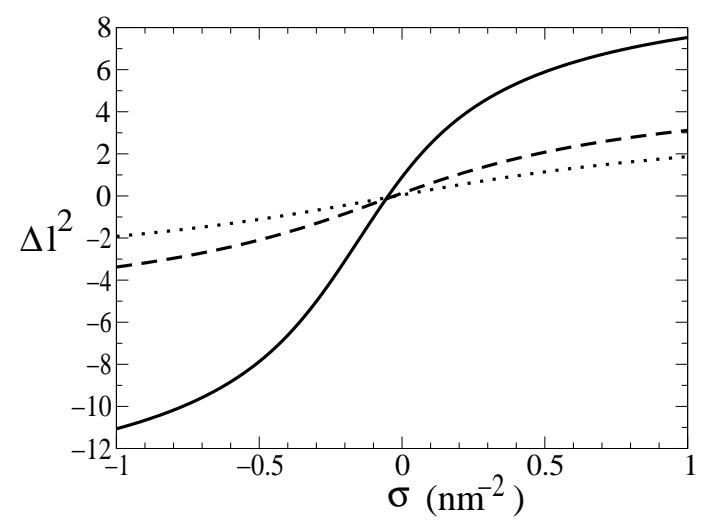

tact with the surface is never more than a couple of $k_{\mathrm{B}} T$, even for quite highly charged surfaces. However, for more modest salt concentrations of $0.1 \mathrm{M}$, rather large free energy decreases per protein molecule are easily obtained at modest charges per unit area on the surface. Also, note that $\Delta$ is slightly positive for an uncharged surface. This is due to the fact that the electrical double layer contribution to the protein-crystal-salt-solution interface is negative, Eq. (22), and this contribution is lost when the protein crystal is brought into contact with the surface.

The potential near the surface, both when the salt solution is in contact with the surface and when the protein crystal is, is shown in Fig. 目 When the protein crystal is in contact with the surface the potential tends to its value in the bulk of the crystal: the Donnan potential, which is 1.48 here. The potential at the surface is larger than one, and so the linear approximation is quite poor for the charge $\sigma= \pm 1 \mathrm{~nm}^{-2}$. In particular, Eq. (24) significantly overestimates the magnitude of $\Delta$ at high surface charges. However, Eq. (24) is reasonably accurate for the smaller charge per unit area, $\sigma= \pm 0.1 \mathrm{~nm}^{-2}$. As an example, for $\sigma=-1 \mathrm{~nm}^{-2}$ and $\rho_{s}=0.1 \mathrm{M}, \Delta l^{2}=-11.1$ without linearisation, whereas Eq. (24) predicts $\Delta l^{2}=-17.0$.

\section{CONCLUSION}

We have used an existing 12, 13] generic model of a protein crystal to estimate the electrostatic contribution $\Delta$ to the free energy change when a protein crystal is brought into contact with a surface with a fixed charge density $\sigma$. Unsurprisingly, the free energy change is negative for a surface with a charge opposite in sign to that on the protein molecule, and positive if the sur- 
FIG. 4: The potential, in units of $k_{\mathrm{B}} T / e$, as a function of distance $z$ from the surface. The solid curves are for a salt solution in contact with the surface and the dashed curves are for a protein crystal against the surface. The upper solid and dashed curves are for a positively charged surface, $\sigma=$ $1 \mathrm{~nm}^{-2}$, and the lower curves are for a negatively charged surface, $\sigma=-1 \mathrm{~nm}^{-2}$. The salt concentration is $0.1 \mathrm{M}$, and the protein is positively charged, $\rho_{p}=0.25 \mathrm{~nm}^{-3}$.

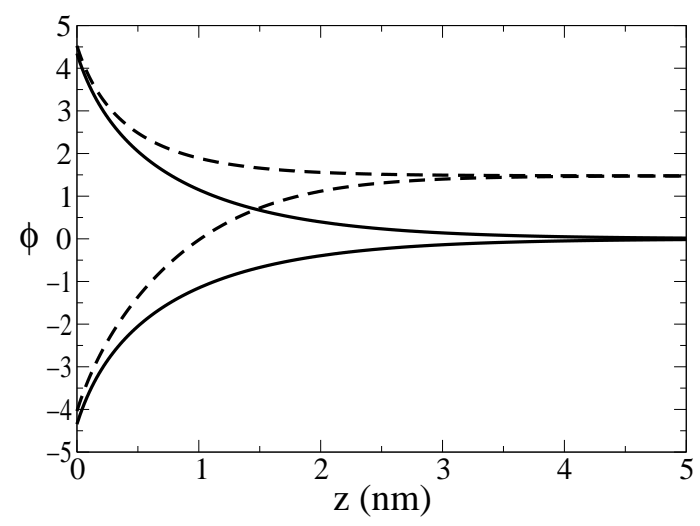

face has a charge with the same sign. We quantified this and found that very reasonable charge densities, of 1 elementary charge per $\mathrm{nm}^{2}$ or less, were sufficient to achieve free energy changes per protein molecule at the surface of several $k_{\mathrm{B}} T$ or more, provided the salt concentration was less than around $1 \mathrm{M}$. The electrostatic contribution to the free energy is the energy associated with the electrostatic interactions in between the surface, protein molecules, and salt and counterions, and the translational entropy of the salt and counterions. Typically, there will also be non-electrostatic interactions of the proteins with the surface, particularly if the surface is at least moderately hydrophobic. Then the proteins may even unfold resulting in irreversible adsorption. Thus, our results will be relevant only to those surfaces which are sufficiently hydrophilic that the proteins interact only weakly with the surface and do not unfold. However, obtaining such surfaces is relatively straightforward via the use of self-assembled monolayers [8, 9]. Our model of the protein is a very simple one, appropriate for proteins with reasonably large net charges, so that the terms we calculate should be dominant, and where this charge is distributed so that the charge density facing the surface is neither much higher nor much lower than the average charge density on the surface of the protein.

Perhaps the most important reason for considering protein crystals at surfaces is interest in their heterogeneous nucleation. Protein crystals nucleate at surfaces and so without an understanding of crystallites at surfaces we cannot hope to understand how the crystals actually form. Crystallising proteins is essential for the determination of their all-important three-dimensional structure via X-ray crystallography 2, 3]. Heterogeneous nucleation is an activated process 1] and so proceeds at a rate which scales as $\exp \left(-\Delta F^{*} / k_{\mathrm{B}} T\right)$, where $\Delta F^{*}$ is the height of the free energy barrier which must be overcome. Typical sizes of the critical nuclei in nucleation are a few 10s of protein molecules, and so one face of a crystalline nucleus has perhaps 10 , or a few less, protein molecules. The critical nucleus is that at the top of the barrier, the nucleus which requires a free energy $\Delta F^{*}$ to create it [1]. Thus, the variation in $\Delta F^{*}$ with $\Delta$, will be approximately $10 \Delta l^{2}$. From Figs. 2 and 3 , we see that this may easily be $10 \mathrm{~s}$ of $k_{\mathrm{B}} T$, causing increases or decreases in the rate of heterogeneous nucleation of many orders of magnitude. The barrier to nucleation may be lowered by $10 \mathrm{~s}$ of $k_{\mathrm{B}} T$, inducing nucleation in solutions which would otherwise be metastable, or it may be raised by 10 s of $k_{\mathrm{B}} T$ preventing nucleation from occurring on one part of the surface. The former is required if a surface is to be used as a nucleant: a surface which triggers nucleation [4, 5]. The latter is required for spatial control of heterogeneous nucleation [6, 7].

Finally, we consider wetting by the crystalline phase of the surface-salt-solution interface. At coexistence, for a bulk solution phase in contact with the surface, a direct surface-salt-solution interface is not the only possibility. It is possible for a slab of the protein crystal to be interposed between the surface and the solution phase. The direct surface-salt-solution interface is then replaced by by a surface-protein-crystal interface plus solution-protein-crystal interface. The protein crystal is said to have wet the surface-salt-solution interface. Wetting occurs at equilibrium whenever it lowers the free energy 14, 22]. Denoting the full interfacial tensions of the surface-salt-solution, surface-protein-crystal and solution-protein-crystal interfaces by $\gamma_{s}, \gamma_{x}$ and $\gamma$, respectively, we have that the free energy change on interposing a slab of protein crystal between the surface and the solution is $-\gamma_{s}+\gamma_{x}+\gamma$. Note that here the interfacial tension of the solution-protein-crystal interface appears with a positive sign whereas it appears with a negative sign in the definition of $\Delta$, Eq. (23). When a layer of the protein crystal is created so is a solution-protein-crystal interface, whereas when an existing protein crystal surrounded by solution is brought into contact with the surface a solution-protein-crystal interface is destroyed.

Determining the sign of $-\gamma_{s}+\gamma_{x}+\gamma$ is not possible without determining both the electrostatic and nonelectrostatic contributions to all three interfacial tensions. However, in Ref. [12], the interfacial tension $\gamma$ was estimated to be a few $k_{\mathrm{B}} T$ per area of a protein molecule, i.e., $\gamma l^{2}=O(1)$. If we further assume that $-\gamma_{s}+\gamma_{x}$ is dominated by electrostatics, then in the linear regime $-\gamma_{s}+\gamma_{x} \simeq \sigma \phi_{\mathrm{D}}$, from Eqs. (9) and (17). Thus for the electrostatic attraction between an oppositely charged surface and a protein crystal to drive wetting of the surface-salt-solution interface, we require that $\left|\sigma \phi_{\mathrm{D}}\right| l^{2}>O(1)$. For highly charged surfaces, $\sigma$ of order $1 \mathrm{~nm}^{-2}$, and low salt concentrations, this inequality is 
easily satisfied.

If the protein crystal wets the surface-salt-solution interface, then assuming that the layer of protein crystal can itself nucleate on the surface it will do so and the bulk protein crystal can grow from this layer at the surface. Then the barrier to crystallisation will be close to zero and the protein will readily crystallise from solution 23. This assumes that the layer itself can nucleate, see Ref. 22] for an introduction to wetting layers, both at equilibrium and their nucleation.

\section{Acknowledgments}

I would like to thank P. Warren for introducing me to the theory of electrostatic effects in proteins. Work supported by The Wellcome Trust (069242).
[1] P. G. Debenedetti, Metastable Liquids (Princeton University Press, Princeton, 1996).

[2] F. Rosenberger, P. G. Vekilov, M. Muschol and B. R. Thomas, J. Cryst. Growth 168, 1 (1996).

[3] N. E. Chayen, Trends Biotech. 20, 98 (2002).

[4] A. McPherson and P. Shlichta, Science 239, 385 (1988).

[5] N. E. Chayen, E. Saridakis, R. El-Bahar and Y. Nemirovsky, J. Mol. Biol. 312, 591 (2001).

[6] A. Sanjoh and T. Tsukihara, J. Cryst. Growth 196, 691 (1999).

[7] A. Sanjoh, T. Tsukihara and S. Gorti, J. Cryst. Growth 232, 618 (2001).

[8] E. Ostuni, L. Yan and G. M. Whitesides, Coll. Surf. B: Biointerfaces 15, 3 (1999).

[9] S. L. Clark, M. F. Montague, and P. T. Hammond, Macromolecules 30, 7237 (1997).

[10] K. Wagner, D. Harries, S. May and V. Kahl, J. O. Rädler and A. Ben-Shaul, Langmuir 16, 303 (2000).

[11] D. Murray, A. Arbuzova, G. Hangyás-Mihyálynyé, A. Gambhir, A. Ben-Tal, B. Honig and S. McLaughlin, Biophys. J. 77, 3176 (1999).

[12] R. P. Sear and P. B. Warren, J. Chem. Phys. 117, 8074 (2002).

[13] P. B. Warren, J. Phys.: Cond. Matt. 14, 7617 (2002).

[14] J. Israelachvili, Intermolecular and Surface forces (Academic Press, London, 1992).

[15] This slightly nonobvious notation is for consistency with Ref. [12].
[16] M. Muschol and F. Rosenberger, J. Chem. Phys. 103, 10424 (1995).

[17] M. Muschol and F. Rosenberger, J. Chem. Phys. 107, 1953 (1997).

[18] B. Guo, S. Kao, H. McDonald, A. Asanov, L. L. Combs and W. W. Wilson, J. Cryst. Growth 196, 424 (1999).

[19] W. C. K. Poon, S. U. Egelhaaf, P. A. Beales, A. Salonen and L. Sawyer, J. Phys.: Cond. Matt. 12, L569 (2000).

[20] S. Sivasankar, S. Subramaniam and D. Leckband, Proc. Nat. Acad. Sci. 95, 12961 (1998).

[21] C. Tanford and R. Roxby, Biochem. 11, 2192 (1972).

[22] D. Bonn and D. Ross, Rep. Prog. Phys. 64, 1085 (2001).

[23] Throughout this work, we consider a protein-solution phase which is sufficiently dilute that it can be considered to be just a salt solution, and only one phase transition: the fluid-crystal phase transition. This crystal phase will wet the surface-salt-solution interface if the surface attracts the protein molecules sufficiently strongly. Some proteins, such as lysozyme exhibit another phase transition, that between dilute and concentrated protein solutions, see Ref. [17]. Wetting can also occur at this second phase transition: the concentrated protein-solution phase may wet the interface between the surface and dilute protein-solution phase. This wetting by a phase other than the crystalline phase, if it occurs, will also strongly effect heterogeneous nucleation of the crystalline phase, see R. P. Sear, J. Phys.: Cond. Matt. 14, 3693 (2002). 